\title{
METHODOLOGICAL APPROACH TO FORMULATE PRODUCTION AND OPERATIONS STRATEGIES IN THE SMES USING THE ANP METHODOLOGY
}

\begin{abstract}
This work aims to develop a methodology based on ANP in order to help formulate strategies must prioritize SMEs, considering two types of strategies: production or operations and thus facilitate the process of decision making and reduce the risk for companies by their nature, are faced with greater force to the problem of resource allocation, thereby achieving an improvement in performance and use of the opportunities offered by the sector in which they operate. For this, a literature review will be done by identifying the main references to address the problems described and subsequently proposed two case studies which are two companies with a focused SMEs in production and the second service delivery model will be applied.
\end{abstract}

Keywords: Strategy, Production, Operation, ANP.

\section{Introduction}

It is understood that the strategy is a project with a series of steps in order to reach a goal in a given time, but most SMEs are not aware of it, so it is interesting to provide this sector of tools to facilitate their decision-making process by increasing knowledge of the real needs and potential to meet them. In his article (Brown, Squire, \& Lewis, 2010), quotes (Tunälv, 1992) who believes that companies with a strategy formulated operation, achieve greater business performance that companies without a strategy of this kind. On the other hand the production strategy is considered as an action plan in the long term that serves to contribute to the overall objectives of the organization, within the scope of their business and corporate strategies. Thus a pattern for proper development of the tactical and operational decisions is obtained.

\section{Literature Review}

Then, by means of the following references, it aims to reach and address the issue mentioned at the beginning of this article.

(Lowson, 2002) The purpose of this article is to provide clear rationale and without distortion to collaborate the development of trading strategies as a field of study. Use, try to clarify the nature of operations strategy: its composition, its nature and purpose for which entails. Thus, it confirms that such strategies are developed through the influences that have market and resources that give shape to these strategies.

(Adamides, 2015) Its purpose grant a micro level. It focuses on the interpretive framework of the strategy of operations, linked and aligned with corporate level strategies to apply and obtain information from these processes manner. It is based on theoretical foundations of social practice and actor-network theory and analysis of the realities organizational training process an operating strategy, including in organizational 
contexts, in a conceptual framework for analyzing production and alignment of the operating strategy that develops.

(De Toni, Filippini, \& Forza, 1992) This paper suggests a conceptual model for operations that can be used to identify opportunities and decisions, and thus gain a competitive advantage in global industries. In addition, this paper considers the operation of the operating system and the four phases of the operation of the value chain (design, purchasing, production and distribution). The study of production strategies coming from globalization is performed by three categories of decision: organization and management, management systems and technologies.

\section{Objectives}

This work aims to investigate and propose a methodology to formulate the strategy of production and operation in the SME sector, through Analytic Network Process (ANP). It is therefore essential to carry out some specific goals such as:

- Study the main characteristics of the production and operation strategies and classify those that primarily affect the SME sector.

- Using Analytic Network Process methodology to address the issues raised.

- To implement the proposal in a case study using the methods mentioned above.

\section{Research Design/Methodology}

To start making the methodology, it has analyzed each of the authors mentioned in this paper, and thus the factors that are critical to the development of SMEs in both the production area and in the field will be determined operating. a list of the factors mentioned by each of the authors was performed, then go discarding those jobs that did not fulfill purpose of providing relevant information for this job.

Then those items that have two or more common factors were considered. Only exception was an author, because it was related to two more statements and thus supports more research information. The selected factors are structured in a model-making under the ANP methodology in order to identify those factors that most influence the selection of a type of strategy in an SME.

Three groups and the four phases of operations, identify an array of twelve areas of opportunities and choices for global strategy.

\section{Data/Model Analysis}

To validate this analysis, it is necessary to resort to small and medium-sized enterprises, and that they give us to know how they operate in the day. It is necessary to investigate and investigate its production and operational area, and based on this, to know how to meet the different needs of their customers so that they remain more consistent and still prefer rather than other organizations.

They were analyzed and compared two companies completely different items. Each of them underwent a brief survey which should evaluate each item, from number 1 to 5 . In the first case study is survey the company to an SME dedicated to the production of food for distribution, this company offers its service to small shops and supermarkets in the capital of Chile, however, also make deliveries in other cities of the coast. The second case considers a company that provides computer services, this SME has extensive experience in financial and social security area, in addition to maintenance of software and IT developments for different companies in the country. 
For both cases, a diagram applied in the "Super Decisions ${ }^{\mathrm{TM}}$ " software using the ANP methodology was performed. The proposed model is applied in cases, but is evaluated differently depending on the weighting each company granted to the items of the previous survey. two sets of criteria that will determine the type of strategy to select defined.

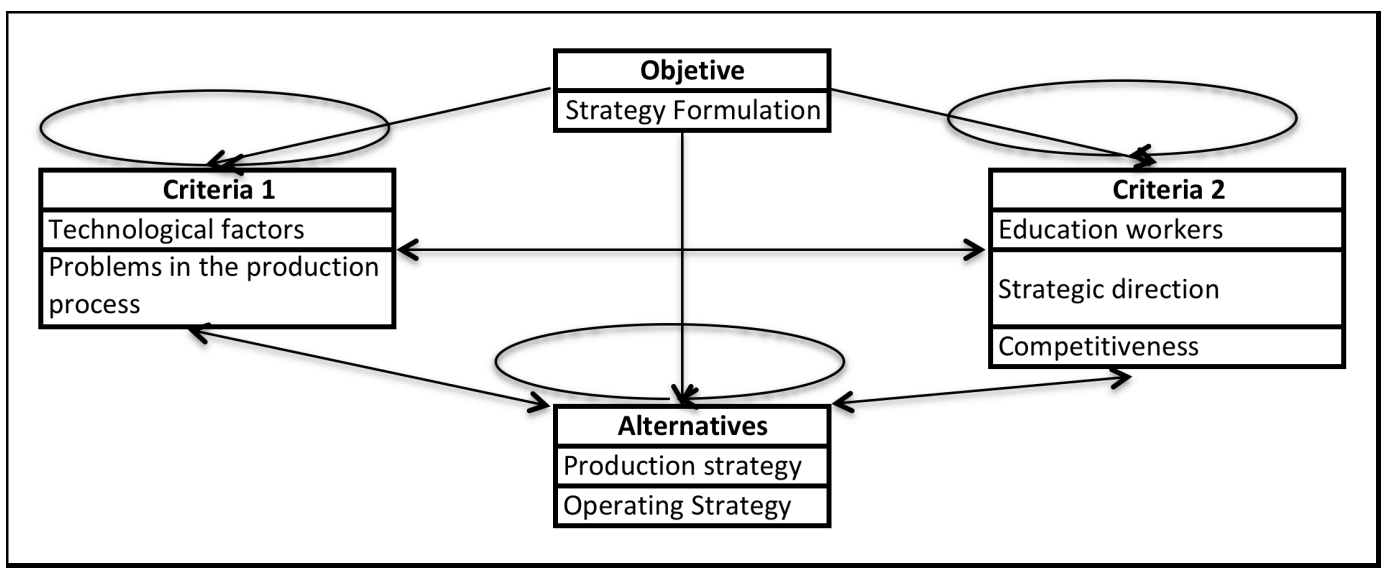

Figure 1: ANP structure

Since the scale of 1 to 9 is used, it must interpolate each element of the survey, since this considered a scale of 1 to 5 for this interpolation the following formula is used:

$$
Y=\frac{\left[\left(X-X_{1}\right)\left(Y_{2}-Y_{1}\right)\right]}{\left(X_{2}-X_{1}\right)}+Y_{1}
$$

Where:

$\mathrm{X}_{1}, \mathrm{Y}_{1}=$ First of coordinates.

$\mathrm{X}_{2}, \mathrm{Y}_{2}=$ Second coordinate.

$\mathrm{X}=$ Coordinate $\mathrm{X}$.

$\mathrm{Y}=$ Interpolating coordinate $\mathrm{Y}$.

In the case of SMEs supply item it shows that the factors that play a role in production, are more important than factors operating area, followed by the factor of strategic direction. He also mentioned at some time that the level of education of its employees has no greater importance, it is why it is the factor with less weight.

\begin{tabular}{|l|r|r|}
\hline \multicolumn{1}{|c|}{ Name } & Normalized by Cluster & Limiting \\
\hline Operating Strategy & 0,336400 & 0,112134 \\
\hline Production Strategy & 0,663600 & 0,221200 \\
\hline Problems in the production process & 0,500000 & 0,166667 \\
\hline Technological factors & 0,500000 & 0,166667 \\
\hline Competitiveness & 0,336230 & 0,112075 \\
\hline Strategic direction & 0,479160 & 0,159719 \\
\hline Education workers & 0,184620 & 0,061539 \\
\hline Stratey Formulation & 0,000000 & 0,000000 \\
\hline
\end{tabular}

Figure 2: Priorities, Food SMEs 
For business services, the most decisive factor is the technology, followed by the level of education of their workers because through these components the company meets the main goal of their work is to develop software by the best professionals.

\begin{tabular}{|l|r|c|}
\hline \multicolumn{1}{|c|}{ Name } & Normalized by Cluster & Limiting \\
\hline Operating Strategy & 0,583330 & 0,194444 \\
\hline Production Strategy & 0,416670 & 0,138889 \\
\hline Problems in the production process & 0,318820 & 0,106275 \\
\hline Technological factors & 0,681180 & 0,227059 \\
\hline Competitiveness & 0,097830 & 0,032611 \\
\hline Strategic direction & 0,449690 & 0,149896 \\
\hline Education workers & 0,452480 & 0,150827 \\
\hline Stratey Formulation & 0,000000 & 0,000000 \\
\hline
\end{tabular}

Figure 3: Priorities, Technology SMEs

In addition, the program provides the weight matrix to proceed to identify the weights of the relations of each node, in this case we obtain the same software. In addition, this Super Matrix weights the importance of the relationship between all nodes of the problem, or in mathematical terms, the relationship between the node 'i' and nodo' $j$ 'are expressed.

\section{Limitations}

Research in this work, limited to the factors of production and operation, because they are very general components. If the components were more specific, they would know more precisely the differences that exist today in SMEs. also you could get more information on each area that makes up the small and medium enterprises, and thus whether there are specific problems that hinder the performance or efficiency of this.

\section{Conclusions}

As could be observed, regardless of the category to develop an SME in our country, technological factors will play a key role in the production environment. This is also because people and industries have increased their use of technology, even in everyday life. Similarly if the technology is to be implemented, this requires prior knowledge, but it is possible that not benefit the industry. The difficulty in the production process, depend on so much experience and management have the organization in terms of its functions. The greater the experience and knowledge of this, the lower the difficulty to achieve the goal. Thus, competitiveness is closely linked to this concept, because there are situations where customers are satisfied with the quality and efficiency of service, causing fidelity to these companies. In these situations, the high command companies maintain calm as they know they are doing an excellent job with its customers. The level of education of employees, each company selects obviously this is due to what the leadership deems appropriate. Along with this factor, the strategic direction is also key, since it is essential to have a vision of what you want and expect in the future the organization. 
ISAHP Article: A Style Guide for Paper Proposals To Be Submitted to the International Symposium on the Analytic Hierarchy Process 2016, London, U.K.

\section{Key References}

Brown, S., Squire, B., \& Lewis, M. (2010). The impact of inclusive and fragmened operations strategy processes on operational performance. International Journal of Production Research. Vol 48, No 14, 4179-4198.

Tunälv, C. (1992). Manufacturing Strategy. Plans and business performance. International Journal of Operations and Production Management, 4-24.

Lowson, R. H. (2002). Operations strategy: genealogy, classification and anatomy. International Journal of Operations \& Production Management, Vol 22 iss: 10, pp.1112.

Adamides, E. D. (2015). Linking operations strategy to the corporate strategy process: a practice perspective. Business Process Management Journal, Vol 21, iss:2, 267-287.

De Toni, A., Filippini, R., \& Forza, C. (1992). Manufacturing Strategy in Global Markets: An Operations Management Model. International Journal of Operations \& Prodution Management, Vol 12 lss: 4, 7-18. 